$r e v i s t a$ docência do ensino

\title{
Avaliação da experiência de estudantes de Odontologia com metodologias ativas de ensino na disciplina de Histologia
}

\author{
Isis Patrícia Soares Silva Dias ${ }^{1}$, Mariana Raquel da Cruz Vegian ${ }^{2}$, \\ Tabata Prado Sato ${ }^{3}$, Keila Cristina Miranda ${ }^{4}$, Luana Marotta Reis de Vasconcellos ${ }^{5}$, \\ Miguel Angel Castillo Salgado ${ }^{6}$, Marianne Spalding ${ }^{7}$
}

\section{RESUMO}

Na busca para aperfeiçoar o processo de ensino-aprendizagem na área da saúde, estratégias e ferramentas pedagógicas que possibilitam uma participação mais ativa dos estudantes têm sido propostas como alternativas para substituir ou complementar o ensino convencional e unidirecional, considerando como pressupostos a aprendizagem significativa, o professor como mediador e a necessidade de aprender a aprender. Nesse contexto, o presente estudo avaliou a percepção de estudantes na disciplina de Histologia, do curso de Odontologia da Universidade Estadual Paulista de São José dos Campos/SP, após a aplicação de estratégias pedagógicas diferentes das aulas expositivas tradicionais. A partir do feedback dos estudantes em relação às experiências vivenciadas em sala de aula, foi possível verificar avaliações positivas quanto à utilização das metodologias ativas de ensino e, embora as opiniões possam divergir em alguns casos, as respostas sinalizam que tais metodologias podem contribuir para uma aprendizagem mais efetiva durante o curso.

Palavras-chave: Educação superior. Educação em Odontologia. Histologia.

\section{Como citar este documento - ABNT}

DIAS, Isis Patrícia Soares Silva et al. Avaliação da experiência de estudantes de Odontologia com metodologias ativas de ensino na disciplina de Histologia. Revista Docência do Ensino Superior, Belo Horizonte, v. 11, e023804, p. 1-17, 2021. DOI: https://doi.org/10.35699/2237-5864.2021.23804.

Recebido em: $16 / 07 / 2020$ Aprovado em: $17 / 12 / 2020$ Publicado em: 21/05/2021

\footnotetext{
${ }^{1}$ Universidade Estadual Paulista “Júlio de Mesquita Filho" (Unesp), São José dos Campos, SP, Brasil. ORCID ID: https://orcid.org/0000-0002-0697-1559. E-mail: isis.patricia@gmail.com

2 Universidade Estadual Paulista “Júlio de Mesquita Filho" (Unesp), São José dos Campos, SP, Brasil. ORCID ID: https://orcid.org/0000-0002-1819-3598. E-mail: mariana.vegian@unesp.br

${ }^{3}$ Universidade Estadual Paulista “Júlio de Mesquita Filho" (Unesp), São José dos Campos, SP, Brasil. ORCID ID: https://orcid.org/0000-0001-6472-8840. E-mail: tabata.sato@unesp.br

4 Universidade Estadual Paulista “Júlio de Mesquita Filho" (Unesp), São José dos Campos, SP, Brasil. ORCID ID: http://orcid.org/0000-0003-1262-821X. E-mail: keila.miranda@unesp.br

${ }^{5}$ Universidade Estadual Paulista “Júlio de Mesquita Filho" (Unesp), São José dos Campos, SP, Brasil. ORCID ID: http://orcid.org/0000-0003-4344-0578. E-mail: luana.marotta@unesp.br

${ }^{6}$ Universidade Estadual Paulista "Júlio de Mesquita Filho" (Unesp), São José dos Campos, SP, Brasil. ORCID ID: http://orcid.org/0000-0001-5793-4013. E-mail: miguel.angel@unesp.br

7 Universidade Estadual Paulista “Júlio de Mesquita Filho" (Unesp), São José dos Campos, SP, Brasil. ORCID ID: https://orcid.org/0000-0003-3667-2434. E-mail: m.spalding@unesp.br
} 


\section{Evaluación de la experiencia de los estudiantes de Odontología con metodologías activas en la disciplina de la Histología}

\section{RESUMEN}

En la búsqueda de mejorar el proceso de enseñanza-aprendizaje en el área de la salud, se han propuesto estrategias y herramientas pedagógicas, que permitan una participación más activa de los estudiantes, como alternativas para reemplazar o complementar la enseñanza convencional y unidireccional, considerando como presupuestos el aprendizaje significativo, el docente como mediador y la necesidad de aprender a aprender. En este contexto, el presente estudio evaluó la percepción de los estudiantes de la disciplina de Histología, del curso de Odontología de la Universidad Estatal Paulista en São José dos Campos/SP, luego de aplicar estrategias pedagógicas diferentes de las clases expositivas tradicionales. A partir de la retroalimentación de los estudiantes sobre las experiencias vividas en el aula, se pudo constatar valoraciones positivas, utilizando metodologías de enseñanza activas y que, si bien las opiniones pueden divergir en algunos casos, las respuestas indican que dichas metodologías pueden contribuir al aprendizaje más efectivo durante el curso.

Palabras clave: Educación superior. Educación Odontológica. Histología.

\section{Evaluation of an experience of Dental Education students with active teaching methodologies in Histology class}

\section{ABSTRACT}

In search of improving the teaching-learning process in the health area, pedagogical strategies and tools, which enable a more active participation of students, have been proposed as alternatives to replace or complement conventional and unidirectional teaching, considering as presuppositions: the meaningful learning; the teacher as a mediator; and the need of learning how to learn. In this context, the present study evaluated the perception of students in Histology class, from the Dentistry degree program at Universidade Estadual Paulista in São José dos Campos/SP, after applying different pedagogical strategies from traditional expository classes. Based on the feedback received from the students about the experiences in the classroom, it was possible to verify positive evaluations, using active teaching methodologies and that, although opinions may diverge in some cases, the answers indicate that such methodologies can contribute to more effective learning during the degree program.

Keywords: Higher education. Dental education. Histology. 


\section{INTRODUÇÃO}

A Histologia, uma das disciplinas geralmente ministradas no início dos cursos da área da saúde, destina-se ao estudo dos aspectos microscópicos, estruturais e funcionais dos tecidos, órgãos e sistemas. Situa-se na encruzilhada entre as ciências médicas macroscópicas e as moleculares, conectando aspectos da anatomia, da bioquímica, da patologia e da fisiologia humana, com o objetivo de promover a base dos conhecimentos necessários para que o aluno compreenda as atuações clínicas (JOHNSON et al., 2015). Apesar disso, muitos estudantes do curso não identificam a relevância da disciplina para sua futura carreira, julgando-a difícil de estudar, especialmente sem prévia experiência com o assunto (JOHNSON et al., 2015).

O uso de recursos digitais para atividades práticas que vão além da utilização de laboratórios de microscopia óptica para observação de lâminas, envolvendo tecnologias da informação e da comunicação, tem sido foco de diversas universidades, ao longo dos anos, para estimular o interesse dos alunos (HEIDGER et al., 2002; KUMAR et al., 2006; SANTA-ROSA; STRUCHINER, 2011). Diante das habilidades tecnológicas e do costume com recursos interativos, as novas gerações não têm demonstrado estímulo para o aprendizado pelo ensino convencional e unidirecional (docente-ativo/estudante-ouvinte-passivo) durante exposições teóricas, mesmo quando se utilizam recursos audiovisuais (SIVIERO; OLIVEIRA, 2016). Segundo Trevisan e Amaral (2016), que desenvolveram um método de avaliação baseado na taxionomia de Bloom, o aluno aprende mais ao decidir (avaliar, escolher, julgar, prever), sintetizar (hipotetizar, criar, formular, integrar) e analisar (comparar, diferenciar, relacionar, debater), habilidades que podem não ser desenvolvidas de forma efetiva quando é utilizado o modelo tradicional de ensino, apenas com aulas expositivas.

Cursos de graduação, inclusive os da área da saúde, como Odontologia, têm sido estimulados a promover reflexões sobre novas perspectivas para a educação. Dentre elas, a elaboração de propostas pedagógicas com metodologias de ensino que permitam capacitar os futuros profissionais de acordo com os novos perfis delineados para eles, com a utilização de metodologias ativas, buscando formas de desenvolver o processo de aprender e de solucionar desafios, em diferentes contextos (BERBEL, 2011).

A busca por diferentes abordagens pedagógicas tem como base as Diretrizes Curriculares Nacionais do Curso de Graduação em Odontologia, publicadas em 2002, que trazem como objetivo do curso habilitar profissionais com competências para resolução do problema de saúde e com capacidade de tomar decisões. O documento cita ainda a importância do desenvolvimento, pelos profissionais, da comunicação e da liderança, com compromisso, responsabilidade, empatia, habilidade para tomada de decisões e capacidade de aprender continuamente, tanto na sua formação quanto na sua prática (BRASIL, 2002, art. 4ํ). 
Dessa forma, ao utilizar novas metodologias, é possível incentivar o processo de ensinoaprendizagem em uma concepção de educação crítico-reflexiva, resultando no envolvimento do educando na busca pelo conhecimento, além de estimular a produção de saberes com base na solução de problemas práticos e complexos observados na realidade dos serviços de saúde (MACEDO et al., 2018). Ademais, de acordo com Mitre et al. (2008), é essencial desenvolver metodologias para a formação de um profissional ativo e apto a aprender constantemente, uma vez que a graduação ocorre durante um período relativamente curto, de alguns anos, enquanto a atividade profissional pode permanecer por décadas, e os conhecimentos e competências transformam-se rapidamente.

Seguindo uma filosofia de ensino centrada no aluno, inúmeras possibilidades podem ser utilizadas, como, por exemplo, a estratégia de sala de aula invertida (flipped-classroom). Alguns dos princípios dessa estratégia proporcionam aos alunos uma oportunidade de ter contato com o conteúdo antes da aula, estimulando a preparação prévia e uma conexão entre atividades dentro e fora da sala de aula (ROTELLAR; CAIN, 2016). Flaherty e Phillips (2015) descrevem que a abordagem pedagógica da sala de aula invertida é vantajosa, pois permite o aprendizado dos alunos em seu próprio ritmo e flexibilidade quando se envolvem com recursos eletrônicos.

Metodologias como Problem Based Learning (PBL) e Team Based Learning (TBL) podem utilizar a problematização como princípio inicial para o desenvolvimento das práticas de ensino-aprendizagem. Na área da saúde, metodologias problematizadoras surgiram na década de 1980, na busca de currículos que melhor definissem o aprendizado dos estudantes e quais habilidades cognitivas e afetivas estavam sendo adquiridas (CYRINO; TORALLES-PEREIRA, 2004). Assim, surgiu a problematização, a partir da qual os estudantes buscam responder a um problema em estudo, detectado na realidade, entendido amplamente, considerando suas possíveis causas e determinantes, que em geral ultrapassam os aspectos técnico-científicos (BERBEL, 1998). Essa estratégia pode ser desenvolvida com a observação da realidade, com base em um tema ou uma unidade de estudo, seguida de reflexão sobre as possíveis causas da existência do problema, buscando informações sobre ele, onde quer que elas se encontrem, o que deverá fornecer elementos para a elaboração, crítica e criativamente, de possíveis soluções (BERBEL, 1998).

A análise de problemas também é uma estratégia da metodologia de estudo de caso, já utilizada em cursos de Direito, Administração, Medicina, entre outros. Nesse caso, o aluno é levado à análise de problemas e à tomada de decisões de acordo com conceitos já estudados (BERBEL, 2011). Essa metodologia é recomendada para que o estudante tenha contato com situações que podem ser encontradas na profissão e passe a analisá-las em diferentes ângulos, antes de tomar uma decisão (BERBEL, 2011). 
Outra metodologia, também já utilizada em diversas áreas, inclusive em cursos da área da saúde, é o TBL, ou Aprendizagem Baseada em Equipes, que foi criado no final da década de 1970 por Larry Michaelsen. O objetivo do TBL é desenvolver habilidades de colaboração por meio de equipes de aprendizagem com tarefas de preparação e aplicação de conceitos, feedback constante e avaliação entre os pares (OLIVEIRA et al., 2018). As etapas, descritas por Bollela et al. (2014) e Michaelsen, Knight e Fink (2002), incluem: preparação individual, com uma orientação de estudo do conteúdo antes da aula; resposta a questões, de forma individual e em grupo; e aplicação de conceitos, momento no qual as equipes devem aplicar conhecimentos para resolver questões apresentadas na forma de cenários-problema relevantes.

Além de diferentes estratégias pedagógicas, várias ferramentas são utilizadas na busca de aulas dinâmicas e mais atrativas para estimular o aprendizado. Dentre elas, estão tecnologias educacionais conhecidas como ambientes virtuais de aprendizagem (AVA), desenvolvidas para apoiar atividades de educação a distância. Esses ambientes oferecem um conjunto de tecnologias de informação e comunicação que permitem a cada participante desenvolver as atividades em seu tempo, espaço e ritmo (RIBEIRO; MENDONÇA, G.; MENDONÇA, A., 2007). Podem ser utilizados em atividades presenciais, semipresenciais ou à distância, facilitando a comunicação e a troca de informações entre os participantes (RIBEIRO; MENDONÇA, G.; MENDONÇA, A., 2007).

Diante disso, este trabalho teve como objetivo avaliar a percepção de estudantes do curso de Odontologia, na disciplina de Histologia, com relação a diversas estratégias pedagógicas e ferramentas educacionais, buscando analisar pontos positivos e possíveis fragilidades desses métodos de ensino, para que estratégias de melhoria do processo de ensino-aprendizagem possam ser alcançadas.

\section{METODOLOGIA DO ESTUDO}

A pesquisa foi realizada na Universidade Estadual Paulista "Júlio de Mesquita Filho" (Unesp), no Instituto de Ciência e Tecnologia de São José dos Campos/SP. O campus conta com salas de aula, laboratórios de microscopia e de informática e clínicas de atendimento ao público, que são, essencialmente, para a atuação dos estudantes do curso de Odontologia. Nestes locais, os alunos precisam desenvolver suas habilidades em resolução de problemas, trabalho em equipe, relações pessoais com pacientes e colocar em prática conhecimentos adquiridos em sala de aula.

A aplicação das metodologias de ensino ocorreu durante o ano de 2016, na disciplina de Histologia, com estudantes dos períodos integral (51 alunos) e noturno (31 alunos). Essa disciplina é anual, ministrada durante o primeiro ano do curso e está dividida em Histologia 
Geral e Bucal. Foram desenvolvidas estratégias pedagógicas utilizando metodologias como: estudo de caso com a dinâmica de grupo "batata-quente", sala de aula invertida (flippedclassroom), Team Based Learning (TBL), problematização, além da utilização do ambiente virtual de aprendizagem, no qual foram incluídos estudos dirigidos à distância e um atlas virtual de histologia.

No final do ano, foi aplicado um questionário para analisar a percepção dos alunos, sendo atribuídos os conceitos ótimo, bom, razoável ou ruim para as estratégias pedagógicas utilizadas, permitindo comentários positivos ou negativos sobre cada atividade. 0 questionário também possibilitou avaliar se os estudantes têm maior facilidade para aprender quando estudam sozinhos ou em grupo e se a preferência de ensino, após o desenvolvimento das atividades, seria o método tradicional ou o uso das metodologias aplicadas. Os resultados foram analisados de acordo com a porcentagem de cada resposta em relação ao total de alunos participantes.

\section{METODOLOGIAS DE ENSINO}

\section{Problematização}

$\mathrm{Na}$ aprendizagem por problematização foram apresentadas diversas situações e problemas clínicos relacionados à odontologia e à área médica no geral. Elas exigiam a investigação de diversos campos e o levantamento de dados a partir de conceitos ainda não explorados em sala de aula, com enfoque sobre o sistema nervoso. Foram apresentados aos estudantes cinco problemas, para os quais precisaram buscar respostas e soluções, durante uma semana, a partir de qualquer meio disponível para consulta, como livros, mídias digitais, artigos impressos ou digitalizados, entre outros. Houve, inclusive, a solicitação do auxílio de docentes de outras disciplinas, devido à multidisciplinaridade da resolução dos problemas.

\section{Estudo de caso com a dinâmica de grupo "batata-quente"}

Após a conclusão do módulo de Histologia Bucal, foi utilizado o estudo de caso, no qual eram apresentados aos alunos diversos casos clínicos simulando situações rotineiras da clínica odontológica, como cirurgias, tratamentos ortodônticos e periodontais, além de casos clínicos envolvendo dentística restauradora, endodontia e prótese. Os estudantes tinham que discutir, do ponto de vista microscópico, os fenômenos biológicos relacionados à disciplina envolvendo cada situação.

Posteriormente à apresentação do caso, foi utilizada a dinâmica da "batata-quente". Os alunos foram dispostos em círculo ao som de uma música enquanto um objeto foi passado de mão em mão no sentido da roda. Quando o som era pausado pelo tutor, quem estava 
com o objeto deveria expor, a partir dos conhecimentos já adquiridos durante o desenvolvimento da disciplina, a explicação dos casos, buscando responder "Por quê?", "Para quê?", "Como?", “Quando?" e "Onde?" sobre a ocorrência de cada situação e, após resolver esses questionamentos, descrever possíveis tratamentos.

Por meio dessa prática procurou-se despertar a curiosidade e correlacionar as situações clínicas com a disciplina de Histologia, que é essencialmente básica e por vezes descontextualizada da prática clínica, instigando os estudantes a discutir cada situação e dando significado ao aprendizado.

\section{Sala de aula invertida}

A estratégia da sala de aula invertida é caracterizada pelo acesso dos estudantes ao conteúdo expositivo e à sua base teórica fora da sala de aula, utilizando tecnologias como vídeos que podem ser vistos pelos alunos antes do encontro em sala. Dessa forma, os estudantes têm uma experiência prévia com a parte teórica e o professor pode aproveitar a aula como momento de reflexão, de crítica, e de colaboração entre os alunos, que podem discutir e interagir, impulsionando o aprendizado de todos (BRANCO; ALVES, 2015).

Neste estudo, essa metodologia foi utilizada durante todo o período de desenvolvimento da disciplina, com a orientação aos alunos de leitura do conteúdo dos livros didáticos antes de frequentarem cada aula para discussão, mesmo antes das aulas expositivas. Durante a primeira aula do ano letivo, que teve por objetivo instruir os alunos quanto às normas da disciplina, os estudantes foram orientados também a assistir a um vídeo em casa para o desenvolvimento da metodologia do TBL.

\section{Team-Based Learning (TBL)}

Criada no final dos anos 1970, essa metodologia envolve estratégias como o gerenciamento de equipes de aprendizagem, tarefas de preparação e aplicação de conceitos, feedback constante e avaliação entre os pares com o objetivo de melhorar a aprendizagem e desenvolver habilidades de trabalho colaborativo (OLIVEIRA et al., 2018).

A sessão de TBL foi realizada em três etapas, mencionadas por Bollela et al. (2014): a) preparação (pré-classe), com estudo individual; b) garantia de preparo (em sala de aula), com teste individual, teste em equipe, apelação e feedback do professor; c) aplicação de conceitos (em sala de aula), com questões no formato de testes de múltipla escolha.

Durante a preparação individual, pelo método da sala de aula invertida, os estudantes foram orientados a assistir ao vídeo "Técnicas Histológicas - Uma Abordagem Prática", do Serviço 
de Produção e Tratamento de Imagem do Instituto Oswaldo Cruz (Fiocruz), como preparação prévia (TÉCNICAS..., 2009).

Na segunda etapa, houve a verificação e a garantia de preparo dos estudantes, com um teste individual de 20 questões de múltipla escolha, envolvendo os conceitos mais relevantes apresentados no vídeo estudado previamente. Os alunos "apostavam" nas respostas certas, ou em mais de uma resposta se estivessem em dúvida, com valores de 0 a 4 pontos, dependendo da certeza, ou não, da escolha. Se estivessem seguros da alternativa correta, atribuiriam 4 pontos nesta alternativa. Se estivessem hesitantes em relação a alguma outra, poderiam distribuir a pontuação entre as alternativas. Por exemplo, 3 pontos para aquela em que tivessem quase certeza e 1 para aquela que acreditavam ser também uma opção, e assim por diante. Toda a pontuação foi anotada em uma folha de respostas (FIGURA 1a).

Ainda nessa etapa, no teste em equipe, o mesmo questionário foi apresentado. Houve a necessidade de discutir as respostas e buscar a alternativa acordada por todos, que deveria ser, então, verificada em uma "raspadinha" (FIGURA 1b). A resposta, supostamente correta, deveria ser raspada. Se estivesse adequada, apresentaria uma estrela, se não, estaria em branco e os alunos teriam que escolher outra. Cada vez que escolhessem a resposta errada antes da certa, a questão teria uma pontuação menor. $O$ acerto na primeira revelação valeria 4 pontos, na segunda 2 pontos, na terceira 1 ponto e na quarta valeria zero. Após os testes apresentados, os alunos realizaram uma apelação referente às respostas com as quais não concordavam, baseando seus argumentos em evidências encontradas em materiais bibliográficos. Ao final dessa etapa, o professor responsável realizou um feedback com comentários e informações relevantes sobre cada questão.

Durante a terceira etapa, na aplicação dos conceitos, seguiram-se os princípios dos $4 \mathrm{Ss}$, mencionados por Bollela et al. (2014). Foram utilizadas questões de múltipla escolha, para o problema principal (Significant), desafiando os estudantes a fazerem interpretações de casos envolvendo situações reais que poderiam encontrar durante sua carreira profissional. 0 mesmo problema (Same) foi recebido por toda a equipe formada para iniciar um debate. Houve, então, a escolha da resposta específica para o problema (Specific), realizada por todos os membros do grupo. Por fim, foi solicitado aos estudantes que realizassem os relatos simultâneos (Simultaneous Report) das alternativas selecionadas para evitar a manifestação de respostas a partir da argumentação de outras equipes. 


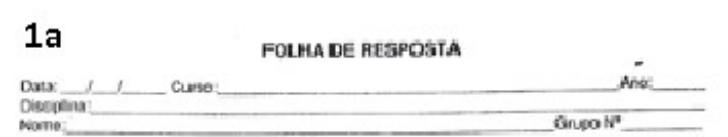

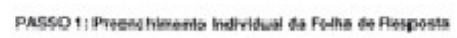

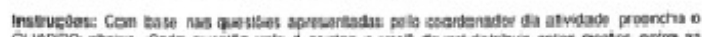

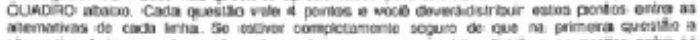

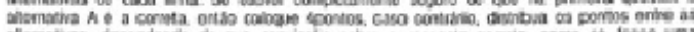

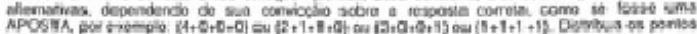

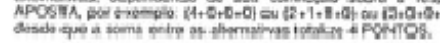

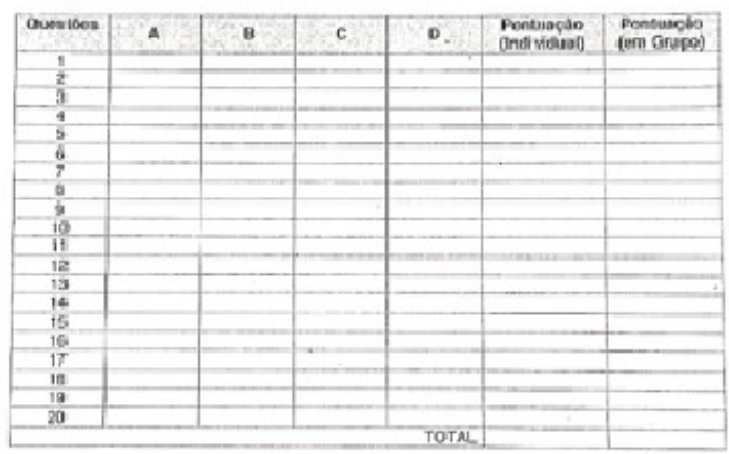

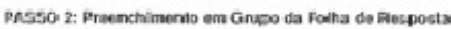

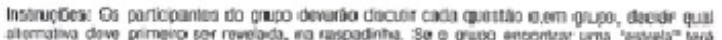

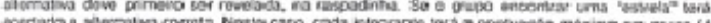

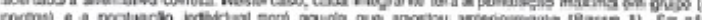

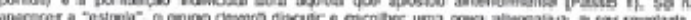

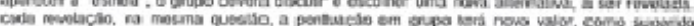

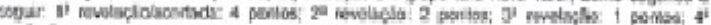

$1 b$

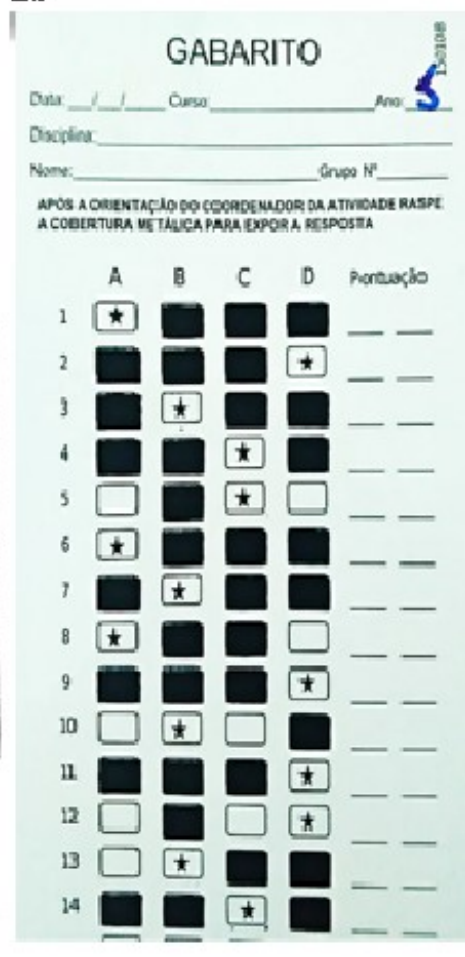

Figura 1 - Questionário e raspadinha utilizados individualmente e em equipes durante a prática de TBL

Fonte: adaptada pelos autores (2016), utilizando modelo do Centro de Estudos e Práticas Pedagógicas da Unesp (CENEPP-Unesp).

A mesma metodologia foi utilizada para a aprendizagem sobre o sistema digestório. Neste caso, o estudo prévio foi realizado durante uma semana com o material didático disponível na biblioteca da universidade.

\section{Ambiente Virtual de Aprendizagem}

No ambiente virtual de aprendizagem, utilizando a plataforma Moodle, foram desenvolvidos questionários, estudos dirigidos e foi disponibilizado um atlas virtual de histologia para facilitar o entendimento dos temas estudados.

Os questionários foram aplicados ao término de todas as aulas da disciplina de Histologia. Os alunos foram divididos em duas turmas: uma delas foi direcionada para o laboratório de microscopia para aulas práticas, comuns durante a disciplina, enquanto a outra turma se dirigiu ao laboratório de informática do Instituto, no qual os estudantes desenvolveram atividades por meio dos questionários relacionados ao conteúdo que foi ministrado naquele dia. Os questionários também podiam ser respondidos por meio de outras mídias digitais que os alunos tivessem à disposição, como celular ou tablet. 
Ainda utilizando a plataforma Moodle, foram realizados estudos dirigidos (FIGURA 2), para que os estudantes pudessem responder à distância, e foi disponibilizado um atlas virtual de histologia, no qual lâminas de cortes histológicos relacionados ao conteúdo ministrado eram disponibilizadas após cada aula prática, para que os alunos tivessem acesso ao material de estudo e pudessem ter contato com ele até o fim do curso.

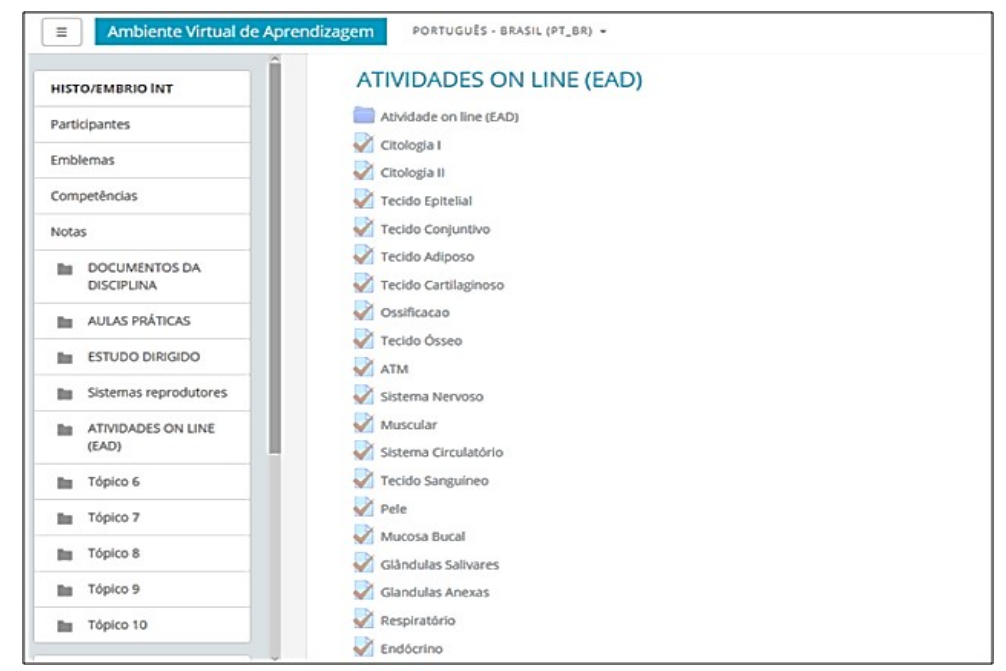

Figura 2 - Questionários hospedados no ambiente virtual de aprendizagem disponibilizados para o estudo dirigido à distância

Fonte: elaborada pelos autores, 2016.

\section{RESULTADOS}

Dos 89 alunos regularmente matriculados na disciplina de Histologia, 82 responderam ao questionário, sendo 51 do período integral e 31 do período noturno. O questionário de avaliação de cada metodologia desenvolvida respondido pelos alunos gerou dados quantitativos mostrados na Figura 3. Em relação a todas as estratégias pedagógicas, mais de $50 \%$ dos estudantes atribuíram conceitos bom ou ótimo.

Na percepção dos alunos, o atlas virtual de histologia, disponibilizado no ambiente virtual de aprendizagem, foi o melhor avaliado, sendo que 93,9\% dos estudantes atribuíram conceitos bom ou ótimo. O TBL também teve uma boa avaliação e $87,8 \%$ consideraram a metodologia boa ou ótima. Com relação à problematização, apenas os alunos do período integral participaram dessa atividade e todos relataram um ou mais aspectos positivos na utilização dessa metodologia, que pode contribuir para a resolução de problemas durante a vida profissional. O estudo de caso com a dinâmica de "batata-quente" também foi avaliado com o conceito ótimo pela maioria dos participantes $(64,5 \%)$.

O estudo dirigido, com questionários para responder em horários diferenciados, foi avaliado como bom ou ótimo por $84,1 \%$ dos estudantes, indicando que o direcionamento de 
atividades para o aprendizado da matéria, após a apresentação do conteúdo desenvolvida em sala de aula, pode ser uma estratégia para consolidação do conhecimento. Contudo, com relação à percepção sobre a melhor forma de aprender, $73 \%$ afirmaram que aprendem melhor estudando sozinhos em comparação com estudar em grupo, demonstrando a preferência ainda pelo método individual de aprendizagem.

No espaço permitido para comentários, alguns pontos negativos foram destacados, como o prazo curto, o formato da apresentação, a dificuldade em compreender os objetivos do trabalho e em encontrar as respostas e soluções para os problemas. No entanto, a maioria dos estudantes preferiu as metodologias e estratégias de ensino aplicadas quando comparadas ao método tradicional de ensino, embora uma parcela de alunos ainda considere o processo tradicional mais eficaz.

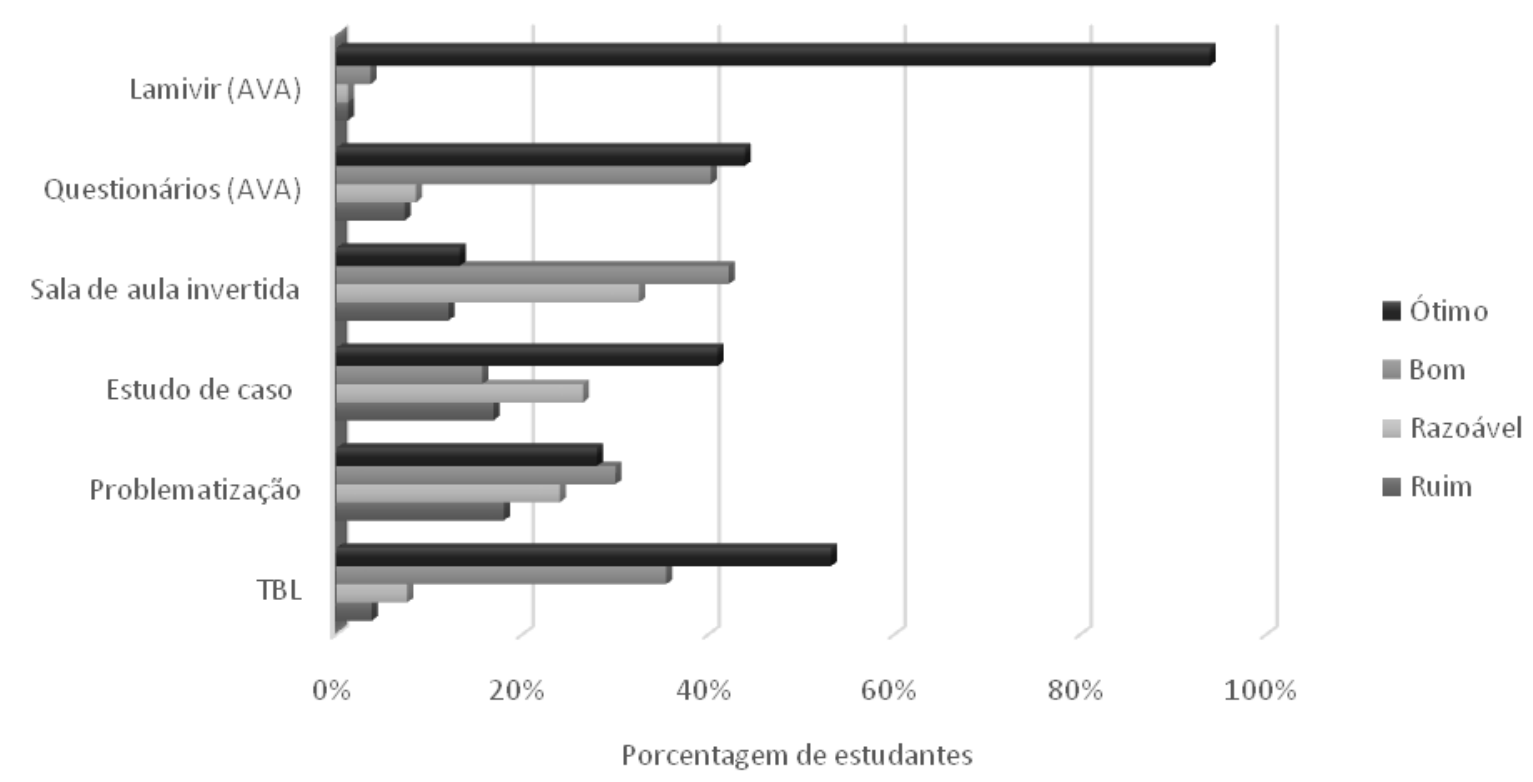

Figura 3 - Gráfico da avaliação dos estudantes sobre a experiência de utilização das ferramentas de ensino-aprendizagem durante a disciplina de Histologia Fonte: elaborada pelos autores, 2016.

\section{DISCUSSÃO}

Diante da complexidade de formar profissionais na área da saúde com métodos que desenvolvam aspectos humanísticos, críticos e reflexivos para sua formação integral, a metodologia tradicional de ensino pode ser insuficiente ou inadequada. Esse cenário tem direcionado uma busca por tendências pedagógicas interativas, que podem estimular o aprender a aprender, com foco nos princípios da pedagogia crítica, reflexiva e transformadora (AZEVEDO; PACHECO; SANTOS, 2019). 
A partir desses princípios, foram utilizadas, neste estudo, estratégias pedagógicas como estudo de caso, problematização, sala de aula invertida, Team-Based Learning (TBL) e atividades em ambiente virtual de aprendizagem - durante a disciplina de Histologia, no curso de Odontologia da Unesp de São José dos Campos/SP - que poderiam contribuir para o desenvolvimento da autonomia e do aprendizado de alunos no ensino superior. A estrutura curricular do primeiro ano do curso de Odontologia inclui disciplinas como a Histologia, para formação inicial e obtenção de bases para os próximos anos, sendo essencial para o aprendizado e o desenvolvimento de caráter crítico durante esse percurso. Dessa forma, a inclusão de metodologias ativas nesse período pode possibilitar um desenvolvimento pessoal e profissional mais significativo.

Após a realização da metodologia de problematização, todos os alunos apresentaram pelo menos um aspecto positivo. A experiência positiva é um importante elemento nessa metodologia, uma vez que ela busca um tratamento reflexivo e crítico dos temas, sendo a realidade social o ponto de partida e de chegada dos estudos do grupo de alunos (BERBEL, 1998). Com a possibilidade de utilização dessa estratégia para o ensino de determinados temas de uma disciplina, devido à especificidade do objeto de estudo, a problematização, que envolve mudanças na programação da disciplina e na postura do professor e dos alunos (BERBEL, 1998), difere-se da metodologia da Aprendizagem Baseado em Problemas (ABP), ou Problem Based Learning ( $\mathrm{PBL}$ ), para a qual é necessário um direcionamento de toda a organização curricular de um curso, com alterações estruturais e integração dos diversos departamentos e disciplinas (CYRINO; TORALLES-PEREIRA, 2004).

Ao desenvolver metodologias como TBL, sala de aula invertida, estudo de caso e problematização, ainda houve comentários negativos e avaliações da experiência de aprendizado com a aplicação das atividades como boa ou razoável. Alguns estudantes apresentam certa resistência ao entrar em contato pela primeira vez com práticas interativas como estas, possivelmente pela cultura da metodologia tradicional, que ainda está presente em grande parte das escolas de ensino regular, e, desse modo, acabam recebendo as inovações de modo passivo. Santa-Rosa e Struchiner (2011) sugerem, após observações de aulas teóricas e práticas e utilização de ambiente virtual para desenvolvimento da disciplina de Histologia e Embriologia, que o aprendizado passivo seja uma característica cultural impregnada no contexto do ensino-aprendizagem, no qual os estudantes recebem de modo passivo o conteúdo transmitido sem que possam questionar, dialogar e compartilhar conhecimentos.

A sala de aula invertida foi sugerida durante todo o curso e aplicada mais efetivamente como metodologia complementar às demais. No entanto, apenas $12,2 \%$ dos estudantes consideraram a estratégia como ótima para o aprendizado. Sua aplicação exige mais disposição do aluno, maior disponibilidade de tempo e apresenta certa complexidade, uma 
vez que há necessidade de interpretar o conteúdo sem o auxílio do professor, demandando mais autonomia e responsabilidade para o aprendizado, além de ação e reflexão na sala de aula (BRANCO; ALVES, 2015). Dessa forma, a necessidade de atitudes diferentes do método de ensino tradicional pode ter causado desconforto e certa resistência pela mudança.

A prática educacional do TBL foi a segunda mais bem avaliada entre os estudantes, obtendo a avaliação ótima para a maior parte dos estudantes. Essa metodologia busca estimular o trabalho em equipe e o desenvolvimento de habilidades de comunicação, argumentação e convencimento, aplicando conhecimentos para resolver questões apresentadas na forma de cenários-problema relevantes (BOLLELA et al., 2014). Uma característica importante dessa metodologia de ensino é o caráter formativo e/ou somativo que reforça a construção da aprendizagem, da responsabilização individual e da importância do reconhecimento de que o sucesso depende de uma boa interação entre os componentes da equipe (BOLLELA et al., 2014). Apesar disso, quando questionados sobre o estudo realizado para consolidação do aprendizado fora da sala de aula, os estudantes informaram a preferência pelo estudo individual (73\%). Em um estudo feito por Johnson et al. (2015) na Universidade de Michigan, também foi verificado que a preferência pelo modo de estudo individual é mais prevalente entre os estudantes dos cursos de Odontologia e de Medicina ao estudar Histologia.

A estratégia avaliada como uma ótima experiência por 90,2\% dos estudantes foi a utilização do atlas virtual de histologia no ambiente virtual de aprendizagem. Com a disposição de lâminas digitalizadas utilizando a plataforma Moodle, os estudantes tiveram contato com materiais que utilizaram durante as aulas práticas de microscopia, o que, provavelmente, facilitou o estudo para atividades avaliativas posteriores. De acordo com Downing (1995) a utilização de imagens digitalizadas permite uma redução expressiva no tempo laboratorial e contribui para a discussão em grupo, geralmente difícil de promover quando os alunos estudam cada um em seu microscópio. A utilização do atlas possibilita o desenvolvimento da autonomia dos estudantes ao facilitar o estudo do conteúdo ministrado em aula prática em períodos determinados por eles mesmos. Esse tipo de ferramenta educacional, de acordo com Santa-Rosa e Struchiner (2011), pode estabelecer relações entre as aulas teóricas e práticas e o estudo extraclasse, buscando despertar o interesse pela Histologia ao tentar diminuir as dificuldades encontradas pelos alunos para o estudo da disciplina.

Apesar de as estratégias pedagógicas serem recebidas, em geral, de forma positiva, comentários negativos foram verificados também durante a avaliação das propostas pedagógicas desenvolvidas, assim como alguns selecionaram a metodologia tradicional como mais eficaz após a experiência com atividades diferenciadas. Essas escolhas, de acordo com Azevedo, Pacheco e Santos (2019), têm como base o pensamento enraizado na educação brasileira e a transição entre os modelos estáveis, consolidados, e os novos, ainda 
em construção, o que caracteriza períodos de inquietação e desconforto e demonstra a complexidade dos desafios da mudança dos métodos de ensino.

\section{CONSIDERAÇÕES FINAIS}

A utilização de metodologias ativas no processo de ensino-aprendizagem, como demonstrado neste estudo, pode ser uma boa alternativa para cursos na área de saúde. É importante ressaltar, no entanto, que os pressupostos pedagógicos primordiais de tais metodologias envolvem uma mudança de concepção na busca por uma aprendizagem significativa, tendo o professor como mediador.

Alguns apontamentos dos estudantes demonstram que a transição do método convencional para metodologias ativas pode causar certo desconforto, pois, neste caso, os alunos precisam sair da "zona de conforto" e desenvolver maior autonomia, passando de meros expectadores para protagonistas no processo de ensino-aprendizagem. Porém, na percepção da maioria dos estudantes da disciplina de Histologia do curso de Odontologia do Instituto de Ciência e Tecnologia da Unesp, as metodologias ativas foram avaliadas de forma positiva, indicando que adaptações e/ou mudanças nas estratégias pedagógicas tradicionais são importantes e podem contribuir para uma aprendizagem efetiva no ensino superior.

\section{REFERÊNCIAS}

AZEVEDO, S. B.; PACHECO, V. A.; SANTOS, E. A. DOS. Metodologias ativas no ensino superior. Revista Docência do Ensino Superior, Belo Horizonte, v. 9, n. 9, p. 1-22, 2019. DOI:

https://doi.org/10.35699/2237-5864.2019.2573.

BERBEL, N. A. N. A problematização e a aprendizagem baseada em problemas: diferentes termos ou diferentes caminhos? Interface (Botucatu), Botucatu, v. 2, n. 2, p. 139-154, 1998. DOI: https://doi.org/10.1590/S1414-32831998000100008.

BERBEL, N. A. N. As metodologias ativas e a promoção da autonomia de estudantes. Ciências Sociais e Humanas, Londrina, v. 32, n. 1, p. 25-40, 2011. DOI:

http://dx.doi.org/10.5433/1679-0383.2011v32n1p25.

BOLLELA, V. R.; SENGER, M. H.; TOURINHO, F. S. V.; AMARAL, E. Aprendizagem baseada em equipes: da teoria à prática. Medicina (Ribeirão Preto), Ribeirão Preto, v. 47, n. 3, p. 293-300, 2014. DOI: https://doi.org/10.11606/issn.2176-7262.v47i3p293-300.

BRANCO, C. C.; ALVES, M. M. Complexidade e sala de aula invertida - considerações sobre o método. In: CONGRESSO NACIONAL DE EDUCAÇÃO, 12, 2015, Curitiba. Anais [...]. Curitiba: Pontifícia Universidade Católica do Paraná, 2015. p. 15464-15477. 
BRASIL. Ministério da Educação. Resolução CNE/CES 3, de 19 de fevereiro de 2002. Institui Diretrizes Curriculares Nacionais do Curso de Graduação em Odontologia. Diário Oficial da União: seção 1, Brasília, DF, p. 10, 2002.

CYRINO, E. G.; TORALLES-PEREIRA, M. L. Trabalhando com estratégias de ensino-aprendizado por descoberta na área da saúde: a problematização e a aprendizagem baseada em problemas. Cadernos de Saúde Pública, Rio de Janeiro, v. 20, n. 3, p. 780-788, 2004. DOI: https://doi.org/10.1590/S0102-311X2004000300015.

DOWNING, S. W. A multimedia-based histology laboratory course: elimination of the traditional microscope laboratory. Medinfo, v. 8, pt. 2, n. 1695, n. p., 1995.

FLAHERTY, J. O.; PHILLIPS, C. The use of flipped classrooms in higher education: a scoping review. The Internet and Higher Education, v. 25, p. 85-95, 2015. DOI: https://doi.org/10.1016/j.iheduc.2015.02.002.

HEIDGER, P. M.; DEE, F.; CONSOER, D.; LEAVEN, T.; DUNCAN, J.; CREITER, C. Integrated approach to teaching and testing in histology with real and virtual imaging. Anatomical Record, v. 269, n. 2, p. 107-112, 2002. DOI: https://doi.org/10.1002/ar.10078.

JOHNSON, S.; PURKISS, J.; HOLADAY, L.; SELVIG, D.; HORTSCH, M. Learning histology - dental and medical students' study strategies. European Journal of Dental Education, v. 19, n. 2, p. 65-73, 2015. DOI: https://doi.org/10.1111/eje.12104.

KUMAR, R. K.; FREEMAN, B.; VELAN, G. M.; DE PERMENTIER, P. J. Integrating histology and histopathology teaching in practical classes using virtual slides. Anatomical Record - Part $B$ New Anatomist, v. 289, n. 4, p. 128-133, 2006. DOI: https://doi.org/10.1002/ar.b.20105.

MACEDO, K. D. S.; ACOSTA, B. S.; SILVA, E. B.; SOUZA, N. S.; BECK, C. L. C.; SILVA, K. K. D. Active learning methodologies: possible paths to innovation in health teaching. Escola Anna Nery, Rio de Janeiro, v. 22, n. 3, p. 1-9, 2018. DOI: https://doi.org/10.1590/2177-9465-ean2017-0435.

MICHAELSEN, L. K.; KNIGHT, A. B.; FINK, L. D. Team-based learning: a transformative use of small groups. Westport: Greenwood publishing group, 2002. Ebook. Disponível em: https://books.google.com.br/books?id=8S8efQkqeqIC\&printsec=frontcover\&hl=ptBR\&source=gbs_ge_summary_r\&cad=0\#v=onepage\&q\&f=false. Acesso em: 26 abr. 2021.

MITRE, S. M.; SIQUEIRA-BATISTA, R; GIRARDI-DE-MENDONÇA, J. M.; MORAIS-PINTO, N. M.; PINTO-PORTO, C.; MOREIRA, T.; HOFFMANN, L. M. A. Metodologias ativas de ensinoaprendizagem na formação profissional em saúde: debates atuais. Ciência e Saúde Coletiva, Rio de Janeiro, v. 13, supl. 2, p. 2133-2144, 2008. DOI: https://doi.org/10.1590/S141381232008000900018.

OLIVEIRA, B. L. C. A.; LIMA, S. F.; RODRIGUES, L. S.; PEREIRA JUNIOR, G. A. Team-Based Learning como forma de aprendizagem colaborativa e Sala de Aula Invertida com 
centralidade nos estudantes no processo ensino-aprendizagem. Revista Brasileira de Educação Médica, Brasília, v. 42, n. 4, p. 86-95, 2018. DOI: https://doi.org/10.1590/1981$52712015 v 42 n 4 r b 20180050$.

RIBEIRO, E. N.; MENDONÇA, G. A. A.; MENDONÇA, A. F. A importância dos ambientes virtuais de aprendizagem na busca de novos domínios da EAD. In: CONGRESSO INTERNACIONAL DE EDUCAÇÃO A DISTÂNCIA, 23, 2007, Curitiba. Anais [...]. Curitiba: Associação Brasileira de Educação a Distância, 2007.

ROTELLAR, C.; CAIN, J. Research, perspectives, and recommendations on implementing the flipped classroom. American Journal of Pharmaceutical Education, v. 80, n. 2, 2016. DOI: https://dx.doi.org/10.5688\%2Fajpe80234.

SANTA-ROSA, J. G.; STRUCHINER, M. Tecnologia educacional no contexto do ensino de histologia: pesquisa e desenvolvimento de um ambiente virtual de ensino. Revista Brasileira de Educação Médica, Rio de Janeiro, v. 35, n. 2, p. 289-298, 2011. DOI:

https://dx.doi.org/10.1590/S0100-55022011000200020.

SIVIERO, F.; OLIVEIRA, S. F. Modernização do ensino de biologia tecidual. Revista da Graduação USP, v. 1, n. 1, p. 35-40, 2016. DOI: https://doi.org/10.11606/issn.2525376X.v1i1p35-40.

TÉCNICAS Histológicas - Uma Abordagem Prática. Produção: Genilton José Vieira, Leonardo Perim, Luzia de Fátima Gonçalves Caputo, Pedro Paulo de Abreu Manso. Roteiro: Luzia de Fátima Gonçalves Caputo, Pedro Paulo de Abreu Manso. Laboratório de Patologia do Instituto Oswaldo Cruz - FIOCRUZ/Serviço de Produção e Tratamento de Imagem do Instituto Oswaldo Cruz - FIOCRUZ. Rio de Janeiro, 2009. 1 vídeo (70 min). Disponível em: https://www.youtube.com/watch?v=RlyTg64AT9E. Acesso em: 27 abr. 2021.

TREVISAN, A. L.; AMARAL, R. G. A Taxionomia revisada de Bloom aplicada à avaliação: um estudo de provas escritas de Matemática. Ciência \& Educação, Bauru, v. 22, n. 2, p. 451-464, 2016. DOI: https://doi.org/10.1590/1516-731320160020011.

\section{Isis Patrícia Soares Silva Dias}

Graduada em Odontologia pela Universidade Estadual Paulista (Unesp) de São José dos Campos, SP, Brasil. Pós-graduanda em Marketing pela Universidade de São Paulo (USP). isis.patricia@gmail.com

\section{Mariana Raquel da Cruz Vegian}

Doutoranda na área de Microbiologia e Imunologia pelo Instituto de Ciência e Tecnologia da Universidade Estadual Paulista (Unesp) de São José dos Campos, SP, Brasil. Mestre na área de Microbiologia e Imunologia pela mesma instituição (2018). Licenciada em Ciências Biológicas pela Unesp de Bauru, SP, Brasil (2014).

mariana.vegian@unesp.br 


\section{Tabata Prado Sato}

Doutora e mestre pelo Programa de Pós-graduação em Odontologia Restauradora, especialidade Prótese Dentária, com período de doutorado sanduíche no exterior na Universidade de Coimbra. Especialista em Saúde Pública. Graduada em Odontologia pela Universidade Estadual Paulista (Unesp) de São José dos Campos, SP, Brasil. Membro do grupo de pesquisa de Inovação em Biomateriais.

tabata.sato@unesp.br

\section{Keila Cristina Miranda}

Residente em Oncologia Molecular no Hospital de Amor de Barretos. Mestre na área de Patologia pela Universidade Estadual Paulista (Unesp) de São José dos Campos, SP, Brasil (2020). Bacharel em Biomedicina pela Universidade Paulista (Unip) (2017), habilitada em Imagenologia, com período sanduíche na The University of Adelaide, SA, Austrália (2013).

keila.miranda@unesp.br

\section{Luana Marotta Reis de Vasconcellos}

Doutora (2005) e mestre (2002) pelo Programa de Pós-graduação em Biopatologia Bucal da Universidade Estadual Paulista (Unesp) de São José dos Campos, SP, Brasil. Docente de Histologia e Embriologia no Instituto de Ciência e Tecnologia da Unesp de São José dos Campos, SP, Brasil. Chefe do departamento de Biociências e Diagnóstico Bucal da mesma instituição.

luana.marotta@unesp.br

\section{Miguel Angel Castillo Salgado}

Doutor em Genética Humana e Médica pela Universidade de São Paulo (USP). Mestre em Biologia e Patologia Buco-dental pela Universidade Estadual de Campinas (Unicamp). Especialista em Morfologia Buco-dental pela Universidade Federal do Rio Grande do Sul (UFRGS). Docente de Histologia e Embriologia no Instituto de Ciência e Tecnologia da Universidade Estadual Paulista (Unesp) de São José dos Campos, SP, Brasil.

miguel.angel@unesp.br

\section{Marianne Spalding}

Doutora (2005) pelo Programa de Pós-graduação em Biopatologia Bucal pela Universidade Estadual Paulista (Unesp) de São José dos Campos, SP, Brasil e mestre (2002) pela Faculdade de Odontologia da Universidade de São Paulo (FOB-USP), Brasil. Docente de Histologia e Embriologia no Instituto de Ciência e Tecnologia da Unesp de São José dos Campos, SP, Brasil, coordenadora do Laboratório de Práticas Pedagógicas (LAPP) e membro do Instituto de Educação e Pesquisa em Práticas Pedagógicas (IEP3) na mesma instituição.

m.spalding@unesp.br 